\title{
Bobina de Helmholtz
}

(The Helmholtz coil)

\author{
Renê Robert \\ Departamento de Eletricidade da UFPR \\ CP 19047, CEP 81531-990, Curitiba, PR
}

Recebido em 24 de outubro, 2002. Aceito em 27 de janeiro, 2003.

\begin{abstract}
É mostrada uma técnica para o cálculo do campo magnético gerado por uma bobina de Helmholtz em torno de seu centro de simetria. São descritos detalhes experimentais e citados experimentos que podem ser realizados com esta bobina, enfatizando que alguns são de custo baixo.
\end{abstract}

It is shown a technique for the calculation of the magnetic field generated by a Helmholtz coil around his symmetry center. They are described experimental details and cites experiments that can be accomplished with this coil, emphasizing that some have low cost.

\section{Introdução}

Freqüentemente é necessário produzir um campo magnético uniforme de baixa intensidade sobre um volume relativamente grande. Para cumprir tal tarefa é, em geral, utilizada a bobina idealizada por Hermann Ludwig Ferdinand von Helmholtz (1821-1894), conhecida atualmente como bobina de Helmholtz, a qual consiste de duas bobinas circulares, planas, cada uma contendo $N$ espiras com correntes fluindo no mesmo sentido conforme esquematizados na figura 1. A separação entre estas bobinas é igual ao raio $R$ comum a ambas. A corrente elétrica de alimentação das bobinas pode ser continua (CC) ou alternada (CA). As aplicações da bobina de Helmholtz são várias; por exemplo: determinação das componentes vertical e horizontal do campo magnético terrestre; anulação em determinado volume do campo magnético terrestre; calibração de medidores de campo magnético de baixa freqüência; estudo dos efeitos de campos magnéticos em componentes ou equipamentos eletrônicos; medidas de susceptibilidade magnética; calibração de equipamentos de navegação; estudo de efeitos biomagnéticos; ajuste de tubos de raios catódicos; estudo da performance de tubos de fotomultiplicadoras em campos magnéticos; medidas de magnetoresistência; desmagnetização de pequenas peças de materiais ferromagnéticos usados na ciência de naves espaciais. $\mathrm{Na}$ área de ensino de física ela é usada principalmente em experimentos para a determinação da carga específica do elétron.

Se as correntes nas bobinas tiverem sentidos opostos, os campos magnéticos gerados por elas terão sentidos opostos. Esta configuração gera um gradiente de campo que é utilizado para o cálculo da força sobre uma amostra material, fato este normalmente usado em balanças de susceptibilidade.

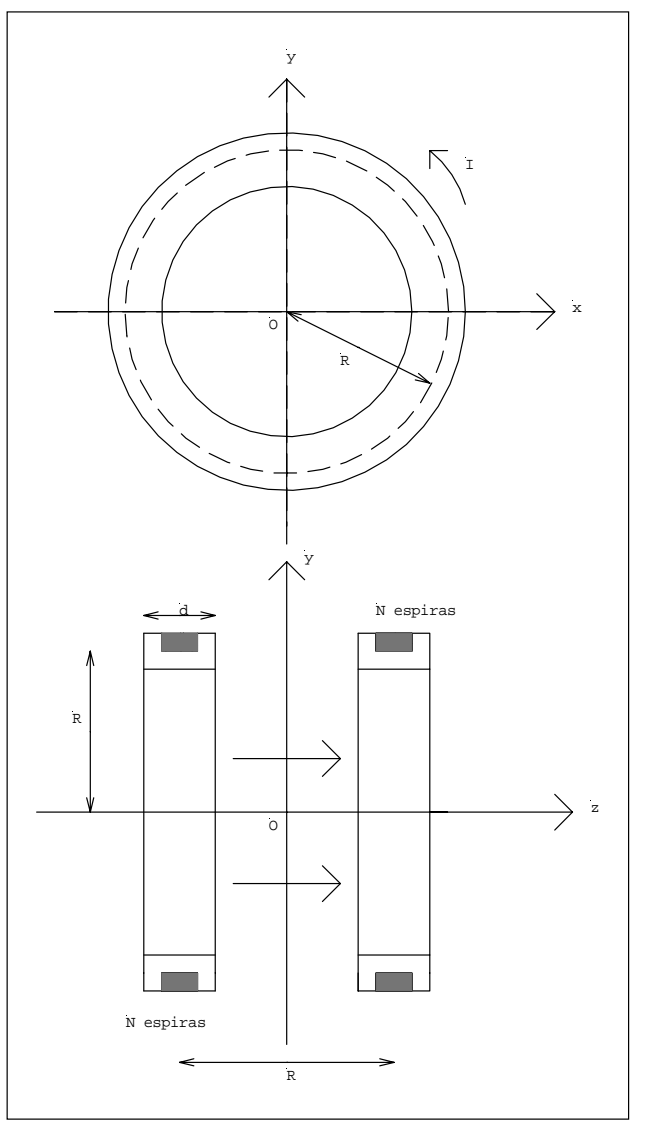

Figura 1. Esquema da bobina de Helmholtz e sua orientação com relação aos eixos oxyz. Nesta bobina em geral fazemos para efeito de cálculo $d \ll R$.

O objetivo deste trabalho é mostrar como calcular o campo magnético no interior da bobina usando o método de expansão axial e incentivar a sua construção e utilização. 


\section{Método de cálculo}

A solução analítica da equação de Laplace para um potencial com simetria axial (potencial eletrostático, magnetostático ou gravitacional) é dada por [1]

$$
V(r, \theta)=\sum_{n=0}^{\infty}\left(A_{n} r^{n}+\frac{B_{n}}{r^{n+1}}\right) P_{n}(\cos \theta),
$$

onde $(r, \theta, \varphi)$ são as coordenadas esféricas de um ponto no referencial cujo eixo $O z$ coincide com o eixo de simetria axial, $P_{n}(\cos \theta)$ é o polinômio de Legendre de $1^{a}$. espécie, $A_{n}$ e $B_{n}$ são constantes.

Para pontos sobre o eixo $O z$ onde $\theta=0$ e $r=z$, a equação (1) se reduz a

$$
V_{\text {eixo }}=\sum_{n=0}^{\infty}\left(A_{n} z^{n}+\frac{B_{n}}{z^{n+1}}\right) .
$$

Existem casos em que o potencial pode ser calculado facilmente ao longo do eixo de simetria $o z$ e que quando expandido em série de potências de $z$, assume a forma

$$
V_{\text {eixo }}=\sum_{n=0}^{\infty}\left(a_{n} z^{n}+\frac{b_{n}}{z^{n+1}}\right) .
$$

Comparando as equações (2) e (3) conclui-se que $A_{n}=$ $a_{n}$ e $B_{n}=b_{n}$, o que permite calcular o potencial $V(r, \theta)$ em regiões não acessíveis à equação (3). Este processo de cálculo é conhecido como "Método da expansão axial" [1,2]. Como regra prática substitui-se em (3) $z^{n} \rightarrow r^{n} P_{n}(\cos \theta)$ e $\frac{1}{z^{n}} \rightarrow \frac{P_{n-1}(\cos \theta)}{r^{n}}$, obtendo-se a solução da equação (1). Nos casos em que a origem $z=0$ pertence ao domínio em estudo somente potencias positivas de $z$ são admissíveis.

Em regiões do espaço onde não existe corrente elétrica, o vetor densidade de corrente $\vec{J}$ é nulo. Conseqüentemente a lei de Ampère se reduz à forma [3] $\vec{\nabla} \times \vec{H}=0$, o que permite escrever $\vec{H}=-\vec{\nabla} V$, sendo $V$ uma função escalar chamada potencial magnético escalar, o qual satisfaz a equação de Laplace.

Por exemplo, para calcular o potencial magnético escalar de uma espira circular de raio $R$ portadora de uma corrente elétrica $I$, parte-se do potencial magnético escalar desta mesma espira calculado em um ponto do eixo $O z$ o qual é dado por [3]

$$
V_{\text {espira }}=\frac{I}{2}\left(1-\frac{z}{\sqrt{R^{2}+z^{2}}}\right)
$$

Desenvolvendo-se a equação (4) em série de potências de $R / z$ ou $z / R$, conforme $z>R$ ou $z<R$, respectivamente, obtem-se após algumas manipulações algébrica as seguinte expressões:

$$
\begin{gathered}
V(r, \theta)=\frac{I R^{2}}{2}\left(\frac{1}{2} \frac{P_{1}(\cos \theta)}{r^{2}}-\frac{1 \times 3 R^{2}}{2 \times 4} \frac{P_{3}(\cos \theta)}{r^{4}}+\ldots\right) \quad z>R, \\
V(r, \theta)=\frac{I}{2}\left(1-\frac{r}{R} P_{1}(\cos \theta)+\frac{r^{3}}{2 R^{3}} P_{3}(\cos \theta)+\ldots\right) \quad z<R .
\end{gathered}
$$

Usualmente a bobina de Helmholtz é constituída de duas bobinas coaxiais de raio $R$, separadas por uma distância $R$, portadoras de mesma corrente elétrica $I$ com mesmo sentido. Cada bobina possui $N$ espiras [4]. As dimensões transversais das bobinas são muito pequenas quando comparadas $\operatorname{com} R(d<<R)$.

Tomando-se a origem do sistema de coordenadas no centro da bobinas de Helmholtz e eixo $O z$ coincidindo com o eixo de simetria axial, obtém-se a seguinte equação para o potencial magnético escalar num ponto deste eixo:

$$
V(z)=\frac{N I}{2}\left(\frac{R / 2-z}{\sqrt{R^{2}+(R / 2-z)^{2}}}-\frac{R / 2+z}{\sqrt{R^{2}+(R / 2+z)^{2}}}\right) .
$$

Expandindo-se a equação (7) para $z<R$ e substituindo-se $z^{n} \rightarrow r^{n} P_{n}(\cos \theta)$, obtém-se

$$
V(\rho, z)=-\frac{8 N I z}{5 \sqrt{5} R}\left(1-\frac{144}{625} \frac{z^{4}}{R^{4}}+\frac{144}{125} \frac{z^{2} \rho^{2}}{R^{4}}-\frac{54}{125} \frac{\rho^{4}}{R^{4}}+\ldots\right),
$$


onde considera-se $\rho^{2}=R^{2}-z^{2}$ e as relações entre as coordenadas cartesianas e esféricas $r^{2}=x^{2}+y^{2}+z^{2} \mathrm{e}$ $\cos \theta=\frac{z}{r}$. Como $\vec{H}=-\vec{\nabla} V(\rho, z)$ obtém-se a componente axial do campo magnético:

$$
H_{z}=\frac{8 N I}{5 \sqrt{5} R}\left(1-\frac{144}{125} \frac{z^{4}}{R^{4}}+\frac{432}{125} \frac{z^{2} \rho^{2}}{R^{4}}-\frac{54}{125} \frac{\rho^{4}}{R^{4}}+\ldots\right)
$$

e também a componente radial:

$$
H_{\rho}=\frac{8 N I}{5 \sqrt{5} R} 72 z \rho\left(\frac{4 z^{2}-3 \rho^{2}+\ldots}{125 R^{4}}\right)
$$

Procedimento semelhante pode ser efetuado quando $z>$ $R$. Outros processos de cálculo podem ser utilizados, por exemplo, o uso da fórmula de Biot-Savart. O cálculo numérico também permite resolver este problema, bem como muitos outros problemas de eletromagnetismo, utilizando diversos softwares comerciais; esta técnica é muito pouco explorada nos atuais livros-texto de eletromagnetismo para físicos.

\section{Cálculo numérico}

Considere-se a seguinte notação:

$$
i=0,1, \ldots 5, \quad j=0, \ldots, 5, \quad H_{0}=\frac{8 N I}{5 \sqrt{5} R}, \quad B_{0}=
$$
$\mu_{0} H_{0}, \quad \frac{i}{10}=\frac{z}{R}, \quad \frac{j}{10}=\frac{\rho}{R} \quad A_{i j}=\frac{H_{z}}{H_{0}}=\frac{B_{z}}{B_{0}}$ a componente de $\vec{B}$ normalizada, na direção axial $O z, B_{i j}=\frac{H_{\rho}}{H_{0}}=$ $\frac{B_{\rho}}{B_{0}}$ a componente de $\vec{B}$ normalizada, na direção radial $O \rho$. Os cálculos foram feitos na aproximação das equações (9) e (10) para $0<\rho<\frac{R}{2}$ e $0<z<\frac{R}{2}$. As matrizes abaixo representam os valores de $A_{i j}$ e $B_{i j}$ calculados em torno da origem com auxílio do software MathCad(B).

$$
\begin{aligned}
& i:=0 . .5 \quad j:=0 \ldots 5 \\
& \begin{array}{c}
A_{i, j}:=1-\frac{144\left(\frac{i}{10}\right)^{4}+432 \cdot\left(\frac{i}{10}\right)^{2} \cdot\left(\frac{j}{10}\right)^{2}-54 \cdot\left(\frac{j}{10}\right)^{4}}{125} \quad B_{i, j}:=72 \cdot i \cdot j \cdot \frac{\left[4 \cdot\left(\frac{i}{10}\right)^{2}-3 \cdot\left(\frac{j}{10}\right)^{2}\right]}{12500} \\
A=\left(\begin{array}{cccccc}
1 & 1 & 1.001 & 1.003 & 1.011 & 1.027 \\
1 & 1 & 0.999 & 1 & 1.005 & 1.018 \\
0.998 & 0.997 & 0.993 & 0.989 & 0.987 & 0.991 \\
0.991 & 0.988 & 0.979 & 0.966 & 0.952 & 0.94 \\
0.971 & 0.965 & 0.949 & 0.924 & 0.893 & 0.859 \\
0.928 & 0.919 & 0.894 & 0.854 & 0.801 & 0.739
\end{array}\right)
\end{array} \\
& B=\left(\begin{array}{cccccc}
0 & 0 & 0 & 0 & 0 & 0 \\
0 & 5.76 \times 10^{-5} & -9.216 \times 10^{-4} & -3.974 \times 10^{-3} & -0.01 & -0.02 \\
0 & 1.498 \times 10^{-3} & 9.216 \times 10^{-4} & -3.802 \times 10^{-3} & -0.015 & -0.034 \\
0 & 5.702 \times 10^{-3} & 8.294 \times 10^{-3} & 4.666 \times 10^{-3} & -8.294 \times 10^{-3} & -0.034 \\
0 & 0.014 & 0.024 & 0.026 & 0.015 & -0.013 \\
0 & 0.028 & 0.051 & 0.063 & 0.06 & 0.036
\end{array}\right)
\end{aligned}
$$

Um exame das matrizes $A$ e $B$ mostra que o campo magnético é praticamente uniforme. Por exemplo, na matriz $A$ observa-se que o campo é constante até a aproximação de $0,1 \%$ na direção $O z(\rho=0)$ para $-\frac{3 R}{10}<z<\frac{3 R}{10}$. O valor máximo do campo magnético ocorre na origem e tem a direção do eixo $O z$. Uma análise semelhante pode ser feita para a componente radial.

As figuras 2 e 3 mostram, numa secção transversal plana $(x=0)$ as superfícies equipotenciais magnéticas e as linhas de campo magnético para os intervalos $-R / 2<z<R / 2$ $\mathrm{e}-R / 2<y<R / 2$. A figura 2 é um modo de representar o lugar geométrico dos pontos onde o potencial magnético $V(\rho, z)=$ const. A figura 3 é outro modo de visualizar o campo $\vec{H}(\rho, z)$, isto é, as linhas onde o campo magnético é tangente em cada ponto. Estas linhas são ortogonais às superfícies equipotenciais. Destas figuras é fácil concluir sobre a uniformidade aparente do campo.

\section{Detalhes construtivos}

Para dimensionar a bobina é preciso fixar, a priori, para quais experimentos ela será usada. A partir da equação $B=\frac{8 \mu_{0} N I}{5 \sqrt{5} R}$ determina-se o raio $R$, a corrente $I$ e o número de espiras $N$ necessário. A bitola do fio a ser utilizado deve ser tal que permita operar a bobina em regime permanente 
sem que o aquecimento, por efeito Joule, danifique o esmalte isolante. A determinação da corrente máxima admissível pode ser feita experimentalmente. A base sobre a qual as bobinas serão fixadas deve ser de madeira ou plástico.

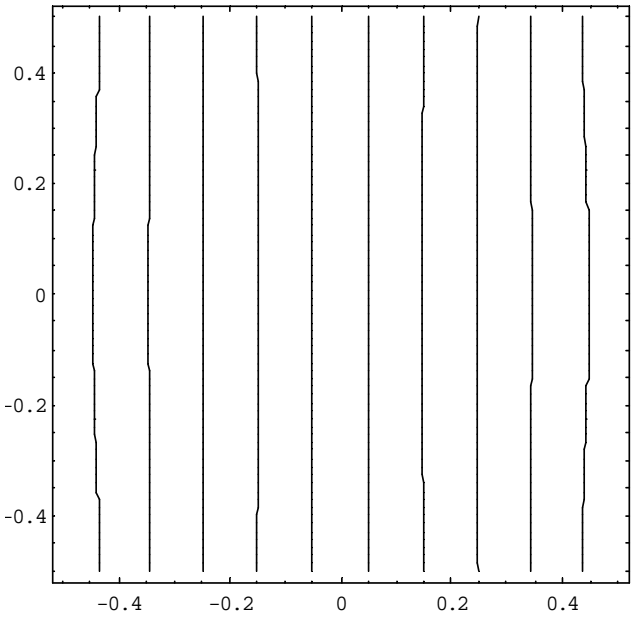

Figura 2. A figura mostra a interseção das superfícies equipotenciais magnéticas com um plano $\varphi=$ const.. As coordenadas são expressas em unidades de $R$, sendo $z$ horizontal e $\rho$ vertical. O gráfico foi feito usando o Mathematica.

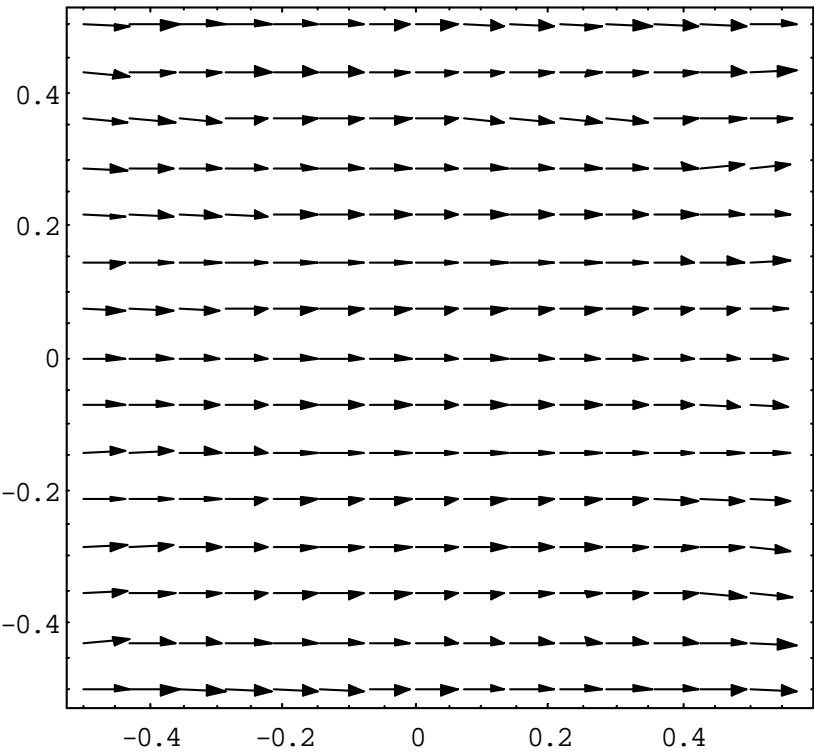

Figura 3. A figura mostra as linhas de campo $\vec{H}$ (ou $\vec{B}$ ) no plano $\varphi=$ const.. As coordenadas são expressas em unidade de $\mathrm{R}$, sendo $z$ horizontal e $\rho$ vertical. O gráfico foi feito usando o Matematica.

As bobinas circulares podem ser montadas em estruturas de alumínio com perfil U calandrado em forma de circunferência. Esta estrutura de alumínio deve ser seccionada quando do uso de corrente alternada, pois desta forma minimizam-se os efeitos de correntes induzidas. Recomenda-se também que a bobina fique o mais afastada possível de paredes, pisos e armários metálicos em particular quando do uso de corrente alternada. Outro elemento indispensável para o uso da bobina é a necessidade de uma fonte $\mathrm{CA}$ ou $\mathrm{CC}$ que permita variar a corrente de alimentação de maneira contínua.

A título de exemplo, a figura 4 mostra uma bobina de Helmholtz que possui $\mathrm{N}=230$ espiras de fio 22 AWG isolado com esmalte e montada em 10 camadas. O raio da bobina é $\mathrm{R}=528 \mathrm{~mm}$. Nesta bobina foi constatado experimentalmente que não é recomendável ultrapassar a corrente de 2,5A quando o uso for prolongado, pois o aquecimento pode comprometer o esmalte do isolamento dos fios. Nestas condições, o campo magnético máximo gerado no centro da bobina é aproximadamente $B_{0}=10$ Gauss. Esta bobina foi construída para a calibração de sensores de campo magnético de $60 \mathrm{~Hz}$ e para a execução de testes de compatibilidade magnética em medidores de energia.

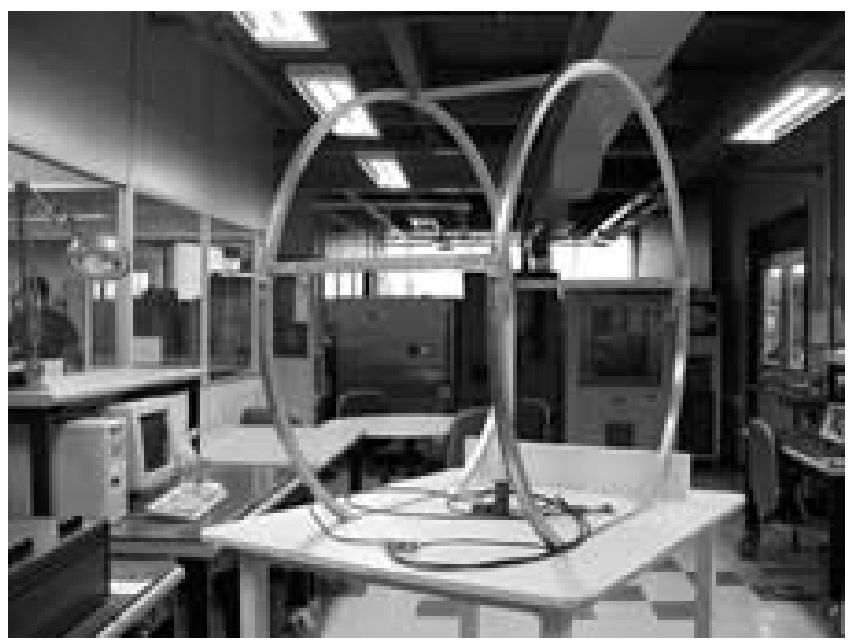

Figura 4. Fotografia de uma bobina Helmholtz com $\mathrm{R}=0,528$ $\mathrm{m}, \mathrm{N}=230$ espiras. A corrente máxima admissível para trabalho contínuo é 2,5 A. O suporte das bobinas são canaletes de alumínio onde se alojam os fios de bitola 25 AWG.

\section{Exemplo de aplicação}

Um exemplo simples de aplicação da bobina de Helmholtz é a determinação da componente horizontal do campo magnético terrestre [5]. Existem diversas maneiras de fazer esta determinação sendo que a mais simples necessita basicamente da bobina de Helmholtz, de uma fonte DC com corrente variável e de uma bússola. A bússola é colocada no centro da bobina, que tem seu eixo orientado na direção norte-sul. Aplica-se uma corrente na bobina de modo a cancelar a componente horizontal do campo magnético terrestre. Quando isto ocorre a agulha da bússola fica em equilíbrio em qualquer direção. Nesta condição, a componente horizontal do campo magnético terrestre vale $B_{0}=$ $\frac{8 \mu_{0} N I}{5 \sqrt{5} R}$. Usando-se a bobina descrita no item IV para a corrente $I=46,8 \pm 0,1 \mathrm{~mA}$ determinada experimentalmente 
obteve-se $B_{0}=0,185 \pm 0,001$ Gauss, o que é o campo que anula o campo magnético terrestre. O método mais comumente utilizado é o método da oscilação de uma pequena barra magnetizada com forma geométrica conhecida, em geral um cilindro, o qual pode oscilar no centro da bobina de Helmoltz. Desta forma pode-se determinar não só a componente horizontal do campo magnético como também o momento de dipolo magnético da barra, desde que se determine o período de oscilação para diversas correntes na bobina.

\section{Conclusão}

A construção de uma bobina de Helmholtz é pouco onerosa e pode, portanto, ser facilmente implementada em qualquer instituição de ensino. Ela se presta desta forma a alguns experimentos tais como: estudos biomagnéticos [5]; determinação da carga especifica do elétron; calibração de bobinas para a medida de campo magnético $\mathrm{CA}$ ou $\mathrm{CC}$; estudo de materiais magneto-resistivos, bem como executar alguns dos experimentos citados na introdução deste trabalho.
A teoria aqui exposta pode ser estendida a outras geometrias com simetria axial ou mesmo resolver outros problemas de eletromagnetismo. Espera-se estar motivando aqueles que têm pendores para resolver problemas com o uso do cálculo numérico a agir, certificando os cálculos com a comprovação experimental.

\section{Referências}

[1] O. D., Jefimenko, Electricity and Magnetism, Electret Scientific Company, Star City, 1989, p.159 e 378.

[2] E., Durand, Électrostatique et Magnétostatique, Masson et Cie Editeurs, Paris, 1953, p.517.

[3] H. M., Nussenzveig, Curso de Física Básica, vol.3 Eletromagnetismo, Editora Edgard Blücher Ltda., São Paulo, 1997, p.143, 152 .

[4] D., Jiles Magnetism and Magnetic Materials, Chapmam \& Hall, London, 1991, p.16.

[5] D., Halliday, R. Resnick and J., Walker, Fundamentals of Physics, John Wiley \& Sons Inc. N.Y., 1993, p.924-938, E101-E10-3. 